\title{
Bayesian Multivariate Mixed-Effects Location Scale Modeling of Longitudinal Relations among Affective Traits, States, and Physical Activity
}

\author{
Donald R. Williams, Siwei Liu, Stephen R. Martin, \& Philippe Rast \\ University of California, Davis
}

\begin{abstract}
Intensive longitudinal studies and experience sampling methods are becoming more common in psychology. While they provide a unique opportunity to ask novel questions about withinperson processes relating to personality, there is a lack of methods specifically built to characterize the interplay between traits and states. We thus introduce a Bayesian multivariate mixed-effects location scale model (M-MELSM). The formulation can simultaneously model both personality traits (the location) and states (the scale) for multivariate data common to personality research. Variables can be included to predict either (or both) the traits and states, in addition to estimating random effects therein. This provides correlations between location and scale random effects, both across and within each outcome, which allows for characterizing relations between any number personality traits and the corresponding states. We take a fully Bayesian approach, not only to make estimation possible, but also because it provides the necessary information for use in psychological applications such as hypothesis testing. To illustrate the model we use data from 194 individuals that provided daily ratings of negative and positive affect, as well as their psychical activity in the form of step counts over 100 consecutive days. We describe the fitted model, where we emphasize, with visualization, the richness of information provided by the M-MELSM. We demonstrate Bayesian hypothesis testing for the correlations between the random effects. We conclude by discussing limitations of the MELSM in general and extensions to the M-MELSM specifically for personality research.
\end{abstract}

Keywords: Personality Assessment, Personality Traits and States, Multivariate Mixed Effect Location Scale Model, Intraindividual Variability, Bayesian Inference

\section{Introduction}

The rise of intensive longitudinal studies and experience sampling methods provide a unique opportunity to ask novel questions about within-person processes relating to personality (Fleeson \& Law, 2015; Vazire \& Sherman, 2017). While personality research has traditionally focused on identifying trait like behavior, for example the degree to which an individual is agreeable, more recent work has broadened its focus to include states (Fleeson, 2001; Martin, Long, \& Poon, 2002). That is, whether, as well as how much, individuals fluctuate in their thoughts, feelings, and behavior over time.

Research on within-person dynamics and short-term fluctuations in behavior has a long tradition in some areas of psy-

Research reported in this publication was supported by funding from the National Science Foundation Graduate Research Fellowship to DRW, the Hellman Foundation to SL, and the National Institute On Aging of the National Institutes of Health under Award Number R01AG050720 to PR. The content is solely the responsibility of the authors and does not necessarily represent the official views of the funding agencies. chology, such as in the field of cognition (Salthouse, 2007), mood (Hepburn \& Eysenck, 1989), and stress reactivity (Sliwinski, Almeida, Smyth, \& Stawski, 2009). Note that the theoretical foundation behind these ideas extend to the realm of personality research. On the macro-time scale it is customary to assume stable personality traits, for example as shown empirically in Costa and McCrae (1988) and CobbClark and Schurer (2012), which relates to an individuals average for some personality trait. On the other hand, on the micro-time scale (e.g., day to day), we might observe substantial variability in these same personality traits (as reported in Fleeson, 2001). The focus here is on within-person variability, that is states, in relation to between-person differences in personality traits. More specifically, it is possible that time-varying predictors moderate the relation between person level traits and fluctuations therein. Of note, the focus is not only on explaining average, between person differences, but also on explaining the observed within-person variance in the respective trait over time or situations.

This conceptualization of personality builds upon a central idea that within-person, or intraindividual variability (IIV), is not regarded as reflecting mere measurement error but 
conveys systematic information (Cattell, Cattell, \& Rhymer, 1947; Fiske \& Rice, 1955; Horn, 1972; Ram \& Gerstorf, 2009; Woodrow, 1932). IIV is commonly indexed by the individual level standard deviation (iS D), wherein an important assumption is that it reflects other aspects of behavioral outcomes compared to individual levels or rates of change, such as, for example, individual means $(i M)$. Indeed, outside of personality research, IIV has been shown to predict cognitive decline, changes in general health and other important life outcomes. For example, IIV has been proposed as a potential marker for Alzheimer's disease (Kalin et al., 2014) and even as a predictor of death (MacDonald, Hultsch, \& Dixon, 2008).

The most common statistical approaches to extracting IIV rely on estimating the individual means ( $i M$ 's) and individual standard deviations (iS D's) using a two-stage approach: In the first stage, the $i M$ 's are computed, for example in a mixed effects model or from individual regressions, and the residuals are recorded. In the second stage, individual SD's are obtained from the residuals which are used in a separate model as either predictor or as the outcome (MacDonald et al., 2008). In the context of personality research this approach was recommended in Eid and Diener (1999) and was recently applied in Hardy and Segerstrom (2017) to characterize the relation between IIV in affect and health. However, this approach suffers from several drawbacks. It can result in unreliable estimates that are particularly sensitive to the number of measurement occasions (Estabrook, Grimm, \& Bowles, 2012; Wang \& Grimm, 2012) and the underlying assumption of normality (Mestdagh et al., 2018; Wang, Hamaker, \& Bergeman, 2012). Moreover, separating $i M$ 's from $i S D$ 's assumes independence of means and variances, which seems unlikely in most applications, and it results in biased variance estimates (Leckie, French, Charlton, $\&$ Browne, 2014; Rast \& Ferrer, 2018).

One aim of the present work is to introduce a Bayesian mixed effects location scale model (MELSM; Hedeker, Mermelstein, \& Demirtas, 2012; Kapur, Li, Blood, \& Hedeker, 2015; Rast, Hofer, \& Sparks, 2012; Williams \& Rast, 2018) for personality research. The MELSM simultaneously estimates sub-models to both personality traits (location) and states (scale) and it accounts for all the underlying covariances among the individual difference parameters in both sub-models. This not only overcomes limitations of the twostage approach, but as we show below, opens the door for rigorously answering novel questions about intraindividual variation in personality.

We are not the first to use a MELSM to study withinperson variability in personality. For example, Hutteman, Back, Geukes, Küfner, and Nestler (2016) used a three-level MELSM to examine variability in personality states across different contexts. Separate models were fitted to several outcomes (e.g., self-esteem and expressive behaviors), with each predicted by aspects of the big 5 inventory. In this paper we present a multivariate model, that is, a MELSM with two outcomes, to model states and traits given 100 daily measures for physical activity and (cross-)lagged autrogressive components in a sample of 194 individuals. To our knowledge, this is the most intensive sampling period that has been used to examine personality traits and states over time, which allows for answering fine grained questions not possible with relatively few observations collected from each individual. Specifically, we investigate the relation between individual differences in positive and negative affect both on average and in IIV conditional on physical activity. We employ Bayesian hypothesis testing to specifically evaluate correlations, or lack thereof (i.e. evidence for the null hypothesis), between individual (random) effects. Finally, our explicit aim is not to address a substantive question, as in Hutteman et al. (2016), but to introduce a general modeling framework that can facilitate the widespread adoption of the MELSM in personality research. This includes $\mathrm{R}$ code for estimating uni- and multivariate MELSM's.

This work is organized as follows. Although our aim is to introduce the multivariate MESLM (M-MELSM), we first provide the rationale for investigating the relations between affect and physical activity. We then introduce the customary MELSM, after which we extend the notation to multivariate data structures. In this section we also emphasize how this model can be used to answer novel questions in personality research, in addition to highlighting the advantages of the presented Bayesian approach. For example, to our knowledge, the full model cannot be estimated with classical methods (e.g., maximum likelihood; Hedeker \& Nordgren, 2013). In the online supplement (link) we present $R$ code for the user-friendly package brms that was used to estimate the reported model (Bürkner, 2017b). The next section focuses on the fitted model, where we emphasize, with visualization, the richness of information that is provided by the Bayesian M-MELSM. We then demonstrate Bayesian hypothesis testing for the correlations between individual (random) effects in particular, as well as describing the inferences that this allows for in practical applications. We end by listing shortcomings as well as possible extensions of the presented model in personality research.

\section{Trait Affectivity and Intraindividual Variability}

While it is customary, in the personality literature, to assess the relations between traditional personality traits and affectivity (Augustine \& Larsen, 2015), for our purposes we focus exclusively on positive and negative affect (denoted PA and NA, respectively). The former is related to positive mood, including feelings of interest, excitement, and enthusiasm, whereas the latter is related to feelings of guilt, nervousness, and distress (Watson, Clark, \& Tellegen, 1988). There is a large body of literature on each construct, and in 
particular, on how they relate to the big five personality inventory (Hutteman et al., 2016; Yik \& Russell, 2001). A well documented finding is that PA is related to extraversion and NA is related to neuroticism (Lucas, Le, \& Dyrenforth, 2008; Wilson \& Gullone, 1999; Wilt, Noftle, Fleeson, \& Spain, 2012). Indeed, PA has been shown to load on the same factor as measures of extraversion (Watson, Clark, McIntyre, \& Hamaker, 1992). On the other hand, a second factor was identified that included indicators of NA and measures of neuroticism (Watson et al., 1992). However, contrary to reflecting the same construct, as argued in Watson et al. (1992), Burger and Caldwell (2000) demonstrated that trait PA was able to explain behavior after accounting for extraversionthe opposite was not the case.

Despite affect and personality typically being treated as related but ultimately different concepts, it is important to note that affect is considered by some as a stable psychological trait (Eid \& Diener, 1999). Moreover, there has been a substantive amount of work on trait affect and health related outcomes, with most of the focus on negative affective styles (Cohen \& Pressman, 2005). For example, NA has been linked to cardiovascular disease (Kubzansky \& Kawachi, 2000; Suls \& Bunde, 2005), immune functioning (KiecoltGlaser, McGuire, Robles, \& Glaser, 2002), and stress reactivity (Chida \& Hamer, 2008). On the other hand, there has been less focus on trait PA. The available evidence points towards an inverse relationships where higher trait levels of PA are associated to lower morbidity, as well as lower self reports of symptoms and pain (for a review see Cohen \& Pressman, 2005).

As noted in Finch, Baranik, Liu, and West (2012), a limitation of the above findings is that PA and NA are typically investigated in isolation of one another. That is, there are few examples that look into both simultaneously, and in particular, the relation between the two over time. This also applies to studies of intraindividual variability. The extant literature is relatively sparse, compared to trait affect, but has been linked to health (Hardy \& Segerstrom, 2017) and aspects of personality (Kuppens, Van Mechelen, Nezlek, Dossche, \& Timmermans, 2007; Timmermans, Van Mechelen, \& Kuppens, 2010). From a substantive perspective, we are, to the best of our knowledge, the first to consider temporal associaitons among PA and NA states. For example, although they were considered in Rast et al. (2012), our model captures the interplay between both by employing a multivariate (i.e., PA and NA are the dependent variables) mixed effects location scale model. Furthermore, by considering PA and NA in the same model, this allows for investigating autoregressive effects on the intraindividual variability across and within the respective constructs. Finally, as an indirect marker of health (Paluska \& Schwenk, 2000; Warburton, Nicol, \& Bredin, 2006), we predict each with physical activity measured with daily step counts. These substantive contributions are novel to the personality literature.

\section{The Mixed Effects Location Scale Model}

To answer the previously described questions the employed model is necessarily complex. Thus, before describing the multivariate MELSM (M-MELSM), we first introduce a simpler, univariate MELSM. This makes the central idea behind the model clear, provides motivation for using it to study personality traits and states, and gives context for the proceeding applied example.

We begin with the standard linear mixed effects model with repeated measurement occasions on $j=1,2, . ., n_{i}$ (occasions)-i.e.,

$$
\mathbf{y}_{i}=\mathbf{X}_{i} \beta+\mathbf{Z}_{i} \mathbf{b}_{i}+\boldsymbol{\epsilon}_{i},
$$

where $\mathbf{y}_{i}$ is a $n_{i} \times 1$ vector of observations for the $i$ th person, $\mathbf{X}_{i}$ is the $n_{i} \times m$ design matrix for the fixed effects of observations for the $i$ th person. Here $\beta$ is a $m \times 1$ vector of fixed effect coefficients. The random effects are in the $n_{i} \times q$ matrix $\mathbf{Z}_{i}$ for observations in person $i$ and $\mathbf{b}_{i}$ is the corresponding $q \times 1$ vector with the random effects coefficients. These effects characterize a person's mean response or location. In the context of personality, this would correspond to an individual's personality trait as measured, for example, by the mean score over time if there is no predictor in the model. $\boldsymbol{\epsilon}_{i}$ is a vector of errors specific to the $i$ th person. In other words, this term corresponds to the fluctuations, or states, around the mean of the respective trait. It is customary to assume that the random effect are distributed as $\mathbf{b}_{i} \sim N(0, \boldsymbol{\Phi})$, where $\boldsymbol{\Phi}$ is a $q \times q$ covariance matrix for the random effects with the variances $\sigma_{b}^{2}$ and covariances $\sigma_{b b^{\prime}}$ for $q \neq q$. The errors are also assumed to be normally distributed with a mean of 0 and covariance of $\sigma_{\epsilon}^{2} \boldsymbol{\Psi}$, where $\boldsymbol{\Psi}$ is a $n \times n$ matrix that can take different structures. For this work, we make the assumption that $\boldsymbol{\Psi}=\boldsymbol{I}_{n}$, wherein $\boldsymbol{I}_{n}$ is a $n$ dimensional identity matrixi.e., the day to day states, or fluctuations around the mean, are assumed to be independent of one another. In these models the between-person variance is captured by $\sigma_{b}^{2}$, whereas the within-person variance is denoted by $\sigma_{\epsilon}^{2}$

\section{Within-Person Variance}

The mixed effects model assumes one value for the error variance $\sigma_{\epsilon}^{2}$, such that, in the context of personality, each individual is assumed to have the same variance in a given trait. The two-stage approach attempts to overcome this by estimating $i S D$ 's for each person. The MELSM, instead, allows $\sigma_{\epsilon}^{2}$ to differ at the individual level-i.e., $\sigma_{\epsilon_{i}}^{2}$. Additionally, we allow it to differ among $j$-time points to obtain $\sigma_{\epsilon_{i j}}^{2}$. Changes in $\sigma_{\epsilon_{i j}}^{2}$ are explained by the time-varying predictors in the $n \times m$ matrix $\mathbf{W}_{i}$ for the fixed effects. The random effects matrix is then $\mathbf{V}_{i}(n \times p ; m \geq p)$ which captures an 

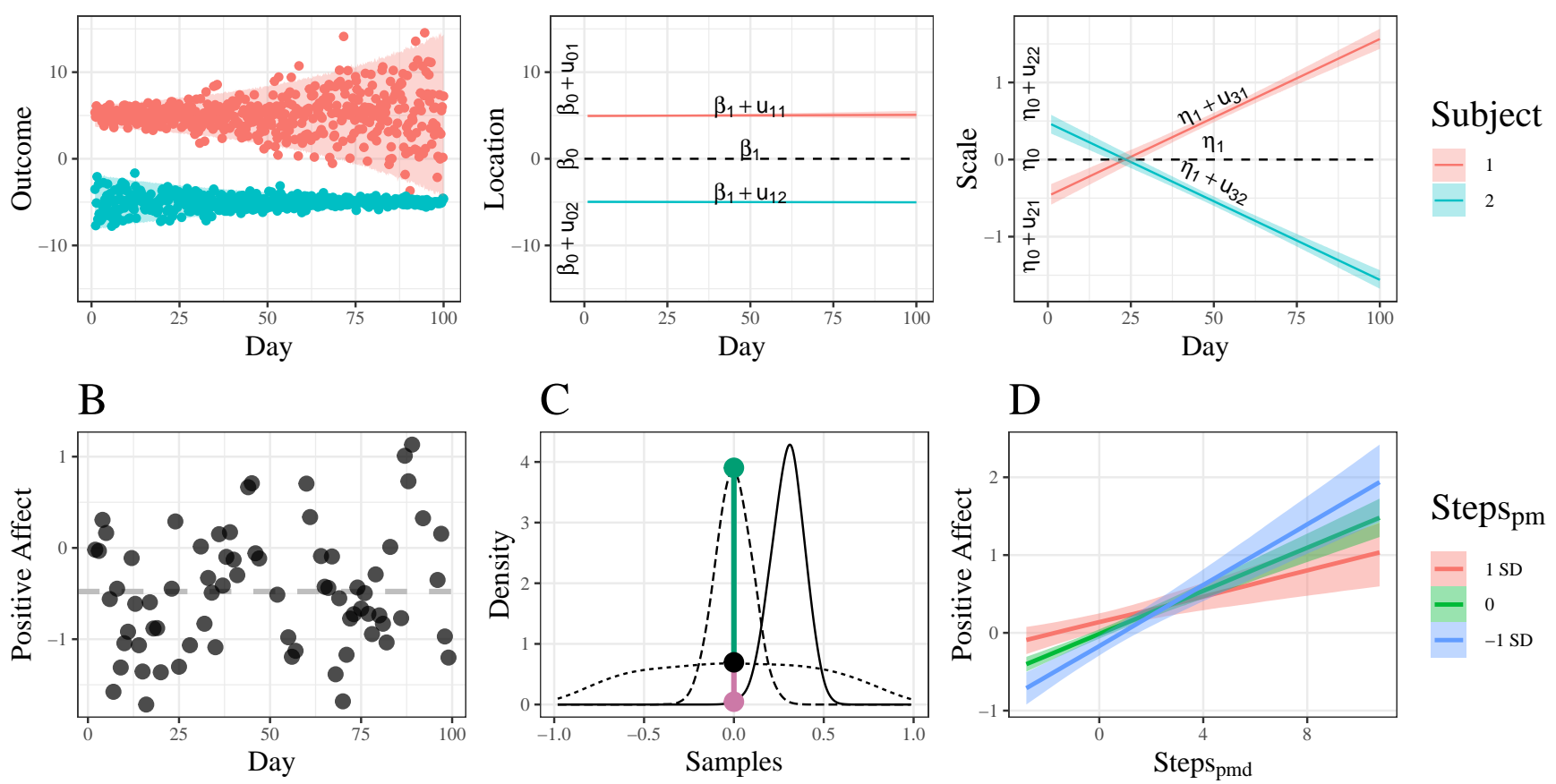

Figure 1. A: An example dataset consisting of raw observations for two individuals (Panel 1), with predicted locations (Panel 2) and log variances (Panel 3) from the MELSM. B: A scatter plot of PA and Day for one subject. The grey line is the person's mean. C: Illustration of Savage-Dickey Bayes Factors for correlations when $H_{0}$ is supported (Green) and when $H_{1}$ is supported (Pink). The dotted line is the implied prior for correlations using the matrix-F distribution. The dashed line is a posterior distribution that favors the null hypothesis $\left(\mathrm{BF}_{01} \approx 5.69\right)$, whereas the solid line is a posterior distribution that supports the alternative hypothesis $\left(\mathrm{BF}_{10} \approx 15.40\right) \mathbf{D}$ : The interaction between mean number of steps and the deviation from the mean on PA. The effect of daily activity on PA is greater for those with less average activity.

individual's variability. Bringing it together, with the inclusion of time-varying covariates, the within-person variance not only varies across persons but also across time given the following model:

$$
\boldsymbol{\varphi}_{i}=\exp \left(\mathbf{W}_{i} \boldsymbol{\eta}+\mathbf{V}_{i} \mathbf{t}_{i}\right)
$$

Note that (2) is for variances, and as such, the exponential function is used to ensure that the estimates are positive real values. $\varphi_{i}$ is a $n \times 1$ vector containing the error variances $\sigma_{\epsilon_{i j}}^{2}$. The fixed effects are denoted with $\boldsymbol{\eta}$ and are analogous to $\boldsymbol{\beta}$ in (1). That is, for an intercept and slope, $\eta_{0}$ is the average within-person variance for a given personality trait and $\eta_{1}$ is effect of some predictor (e.g., time) on the log scale. The individual deviations from these fixed effects are denoted by $\mathbf{t}_{i}$ and are assumed to be normally distributed-i.e., $\mathbf{t}_{i} \sim N(\mathbf{0}, \boldsymbol{\Theta})$. Here $\boldsymbol{\Theta}$ is a covariance matrix of dimensions $p \times p$. Importantly, even with a personality trait that is constant over time, there could nonetheless be effects on the variance and individual variation therein. The MELSM allows for investigating this possibility. This has implications for the study of personality development (for example), in that the focus can be expanded beyond the trait level to also consider variability over time: do fluctuations in personality diminish or increase as a function of an external variable across the lifespan? Or, is variability stable over time and could it be conceived as a trait?

\section{Motivating Example}

Figure 1 illustrates different possible outcomes from a MELSM. In this artificial example we discuss two individuals, each of which provided daily measurements over 100 days (represented as dots in panel A). This hypothetical model is defined as

$$
\begin{aligned}
y_{i j} & =\beta_{0}+\beta_{1}\left(\operatorname{Day}_{i j}\right)+u_{0 i}+u_{1 i}\left(\operatorname{Day}_{i j}\right)+\epsilon_{i j}, \\
\sigma_{i j}^{2} & =\exp \left[\eta_{0}+\eta_{1}\left(\operatorname{Day}_{i j}\right)+u_{2 i}+u_{3 i}\left(\operatorname{Day}_{i j}\right)\right] .
\end{aligned}
$$

This includes four fixed effects, the intercepts $\left(\beta_{0}\right.$ and $\left.\eta_{0}\right)$ and slopes $\left(\beta_{1}\right.$ and $\left.\eta_{1}\right)$, as well as individual deviations, the random intercepts $\left(u_{0 i}\right.$ and $\left.u_{2 i}\right)$ and random slopes $\left(u_{1 i}\right.$ and $\left.u_{3 i}\right)$. As is evident from Panel A, the individual represented by red dots seems to get more variable over time while the withinperson variability for the individual represented by teal dots reduces over time. The location effects are captured in the 
middel of panel $\mathrm{A}$, where the black line, at day 0 , is the fixed effect intercept $\beta_{0}$-the average of these two individuals. Further, $\beta_{0}+u_{01}$ would be the intercept for the first subject. In this example, there would be no effect of day (i.e., $\beta_{1}=0$ ) on the given trait, in addition to no individual variation therein $\sigma_{u 1}^{2}=0$. The scale effects are provided in right most plot of panel A. In reference to (2), the fixed effect intercept $\eta_{0}$ is $\exp (0)=1$. Note that the random intercepts indicate that the individual variance differed on the first day of the study. Furthermore, while on average there is no change in variance over time $\left(\eta_{1}=0\right)$, there are individual differences to consider. One person became more variable over time, whereas the other became less variable over the course of the study. Characterizing these effects, for both the location and scale, is the central idea behind the MELSM. This example illustrates how predictors can influence the location and the scale parameters differentially. In that sense, the MELSM seems ideally suited to capture traits and states in one model while allowing a set of variables to predict both the location and scale parameters.

\section{A Multivariate Mixed Effects Location Scale Model}

\section{Illustrative Data}

We draw data from the iFit study, a research project on daily health behaviors and physical health outcomes. Onehundred-and-ninety-four participants were recruited from a commercial weight loss program as well as from the general population in Sacramento and Yolo counties in California, US. Their ages at recruitment ranged from 20 to 74 years $(\mathrm{M}=40.72, \mathrm{SD}=12.38)$. $71 \%$ percent of the participants were females. $60 \%$ percent were white/Caucasian, $17 \%$ percent were Hispanic, $13 \%$ percent were Asian, and $6 \%$ percent were black/African Americans. Upon providing informed consent, participants completed a set of questionnaires containing demographic and other health-related information. They were then given a Fitbit Charge and were asked to wear it 24 hours a day for at least 100 days. The Fitbit Charge automatically tracked their physical activity in the form of daily step counts. In addition, every evening during the 100 days, participants received a link to an online survey which contained questions regarding their affect, stress, and food consumption. Daily affect was measured using the Positive and Negative Affect Scale (PANAS; Watson et al., 1988), which contained 10 items on positive affect (e.g., attentive, active, excited) and 10 items on negative affect (e.g., hostile, irritable, ashamed). All items were rated on a visual analogue scale from 1 (not at all) to 100 (extremely). The order of the items was randomized across days and persons to minimize carry-over effects. On average, the participants completed 82.46 daily surveys $(\mathrm{Mdn}=93 ; \mathrm{SD}=22.65)$. Items were combined as sum scores which resulted in the two outcomes. Figure 1B depicts a scatter plot of PA across time for one subject of PA.

\section{Model Specification}

The standard multivariate mixed model formulation is described in Maccallum, Kim, William, and Kiecolt-Glaser (1997) and Goldstein (2011). In that formulation the dependent variables are combined into one vector, and then dummy coded variables are introduced to "switch" on the respective outcome. This effectively allows for estimating a multivariate model with a univariate expression. While this is a common "trick" to overcome the limitations of standard software packages that only take univariate outcome vectors such as SAS's proc mixed or R's lme4, it is not necessary for matrix oriented programs such as Stan, and wrapper packages such as brms. Hence, the univariate MELSM can be extended easily to a multivariate model. While, so far, $\mathbf{y}$ was a $n \times 1$ vector for one outcome variable, we can now represent the multivariate outcome in a $n \times k$ matrix $\mathbf{Y}=\left(\boldsymbol{y}^{(1)}, \ldots, \boldsymbol{y}^{(k)}\right)$, where $k$ is the number of dependent variables under consideration. Our dependent variables are PA and NA $(k=2)$, which are assumed to be random variables from a multivariate normal distribution with the mean vector $\boldsymbol{\mu}=\left(\mu_{\mathrm{PA}}, \mu_{\mathrm{NA}}\right)^{\prime}$ and the $k \times k$ covariance matrix $\boldsymbol{\Sigma}$-i.e.,

$$
\mathbf{Y} \sim N(\boldsymbol{\mu}, \boldsymbol{\Sigma})
$$

Note that the residual variances and the covariance among PA and NA are captured in the diagonal and off-diagonal elements of $\boldsymbol{\Sigma}$, respectively. In order to facilitate computation and the definition of priors, we re-expressed $\boldsymbol{\Sigma}$ as $\boldsymbol{\tau} \boldsymbol{\Omega} \boldsymbol{\tau}^{\prime}$, where $\boldsymbol{\Omega}$ is a $2 \times 2$ correlation matrix and $\tau$ is the $2 \times 2$ diagonal matrix of residual standard deviations $\operatorname{diag}(\boldsymbol{\tau})=\boldsymbol{\sigma}=\left(\sigma_{\mathrm{PA}}, \sigma_{\mathrm{NA}}\right)^{\prime}$. The assumed prior distribution for the correlations is

$$
\mathbf{\Omega} \sim \operatorname{LKJ} \operatorname{corr}(v=1),
$$

where LJKcorr is the Lewandowski, Kurowicka, and Joe prior (Lewandowski, Kurowicka, \& Joe, 2009). This distribution is governed by a single parameter $v$. A value of one places a uniform prior over all correlation matrices. This results in a uniform (marginal) prior for the residual correlation that is between -1 and 1 , assuming a $2 \times 2$ matrix. This formulation extends to any number of dependent variables.

Location Model. Before defining the location submodel, we first re-iterate our primary substantive aim. We are primarily interested in the relation between affect and physical activity, including individual variation therein. For time series data it is customary to include a lagged predictor. This not only accounts for the previous days rating, but in the present model, allows for investigating additional questions about longitudinal relations between PA and NA. 
We now define the location sub-model for each person $i$ and day $j$ as

$$
\begin{aligned}
y_{i j}^{(k)}=\beta_{0}^{(k)} & +\beta_{1}^{(k)}\left(\operatorname{Day}_{i j}\right)+\beta_{2}^{(k)}\left(\mathrm{PA}_{\mathrm{Lag} 1, i j}\right)+\beta_{3}^{(k)}\left(\mathrm{NA}_{\mathrm{Lag} 1, i j}\right) \\
& +\beta_{4}^{(k)}\left(\operatorname{Steps}_{\mathrm{pm}, i}\right)+\beta_{5}^{(k)}\left(\operatorname{Steps}_{\mathrm{pmd}, i j}\right) \\
& +\beta_{6}^{(k)}\left(\operatorname{Steps}_{\mathrm{pm}, i} \times \operatorname{Steps}_{\mathrm{pmd}, i j}\right) \\
& +u_{0 i}^{(k)}+u_{1 i}^{(k)}\left(\operatorname{Day}_{i j}\right)+u_{2 i}^{(k)}\left(\operatorname{Steps}_{\mathrm{pmd}, i j}\right)+\epsilon_{i j}^{(k)} .
\end{aligned}
$$

The $(k)$ superscript denotes the column in the matrix $\mathbf{Y}$ and the column in the row vectors $\beta$ that correspond to either PA or NA. Note that both outcomes were standardized to $z$ scores. The predictors include the day elapsed since the beginning of the study (Day), each person's PA and NA rating on the previous day $\left(\mathrm{PA}_{\mathrm{Lag} 1}\right.$ and $\left.\mathrm{NA}_{\mathrm{Lag} 1}\right)$ and the count of daily steps taken. The daily step counts were separated into two components: Each persons average step count across the study $\left(\mathrm{Steps}_{\mathrm{pm}}\right)$ and each persons daily deviation from its average mean $\left(\mathrm{Steps}_{\mathrm{pmd}}\right)$. Note that, when dealing with time-varying variables and autoregressive components, it is important to separate between person level components from time-varying within-person components in order to minimize bias in the parameter estimates (Hamaker \& Grasman, 2015; Wang \& Maxwell, 2015). We also included the interaction $\left(\mathrm{Steps}_{\mathrm{pm}} \times\right.$ Steps $\left._{\mathrm{pmd}}\right)$ to elucidate the interplay between deviations from their average level and their respective average. In other words, it could be that people who are relatively inactive (or active) may react more strongly to deviating from their daily routines. We also considered random intercepts $\left(u_{0 i}\right)$ that provide each person's predicted affect on day 1 , random slopes $\left(u_{1 i}\right)$ for Day that capture individual variation in affect over the course of the 100 days, as well as random slopes $\left(u_{2 i}\right)$ for the deviations from each person's average step count.

Scale Model. The assumed scaled model is similar to that of the location, but with a slightly simplified random effects structure

$$
\begin{aligned}
\sigma_{i j}^{2(k)}=\exp \left[\eta_{0}^{(k)}\right. & +\eta_{1}^{(k)}\left(\mathrm{PA}_{\mathrm{Lag} 1, i j}\right)+\eta_{2}^{(k)}\left(\mathrm{NA}_{\mathrm{Lag} 1, i j}\right) \\
& +\eta_{3}^{(k)}\left(\mathrm{Steps}_{\mathrm{pm}, i}\right)+\eta_{4}^{(k)}\left(\mathrm{Steps}_{\mathrm{pmd}, i j}\right) \\
& +\eta_{5}^{(k)}\left(\mathrm{Steps}_{\mathrm{pm}, i} \times \mathrm{Steps}_{\mathrm{pmd}, i j}\right) \\
& +u_{3 i}^{(k)}+u_{4 i}^{(k)}\left(\mathrm{Steps}_{\left.\left.\mathrm{pmd}_{, i j}\right)\right] .}\right.
\end{aligned}
$$

The predictors for the scale part are the same as in Equation (6). The rationale for including negative and positive affects comes from previous work. For example, negative affect has been shown to influence the variability of positive affect, for both the location (e.g. Röcke, Li, \& Smith, 2009) and scale (e.g. Rast \& Ferrer, 2018). The latter was in the context of dyadic interactions where the partners NA was used to predict the variance in the other partners PA on the same day. The present model extends this notion as it allows for assessing whether the previous days rating of PA or NA influences the following days fluctuation around their respective mean. The random effects in Equation (7) provide each person's deviation from the overall variance at the beginning of the study $\left(u_{3 i}\right)$ and the random slopes $\left(u_{4 i}\right)$ capture individual departures from the average change in variability associated to daily changes in steps taken. The latter allows for answering whether deviations from the typical day explain variability in PA and NA and whether there are individual differences in these effects. Note that for both the location and scale fixed effects we assumed improper prior distributions. We acknowledge this is less than ideal but was done to simplify the model formulation. This is addressed further in the discussion.

\section{Random Effects Variance Model}

An important aspect of the present model is that it allows for estimating correlations between the random effects. Each outcome has 5 random effects in total, and rather than assume separate distributions for PA and NA, we instead estimate a $10 \times 10$ covariance matrix-i.e.,

$$
\boldsymbol{u}_{i} \sim N(\mathbf{0}, \boldsymbol{\Theta})
$$

Here $\boldsymbol{\Theta}$ contains the variances of the random effects of the location and the scale, as well as all covariances, for both PA and NA. We are particularly interested in the covariances, because they capture the interplay, within and between dependent variables, and among location and scale (random) effects. We thus used the matrix- $F$ prior distribution for $\boldsymbol{\Theta}$ which follows

$$
\boldsymbol{\Theta} \sim F\left(v=10, \delta=6, \mathbf{B}=0.4 \boldsymbol{I}_{10}\right),
$$

where $\mathbf{B}$ is a scale matrix and $\boldsymbol{I}_{10}$ a $10 \times 10$ identity matrix. Technical details for this prior distribution can be found in Mulder and Raúl Pericchi (2018), with psychological applications provided in Williams and Mulder (2019) and Williams, Rast, Pericchi, and Mulder (2019). For the present purposes it suffices to note that the parameters (e.g., v) were chosen to reflect a plausible effect size for the implied correlations (Figure 1; panel C), which allows for Bayesian hypothesis testing. Further details are provided in the following section.

Hypothesis Testing. We test for the presence or absence of a correlation by comparing an equality constrained (null) hypothesis versus an unconstrained hypothesis-i.e.,

$$
\begin{aligned}
& \mathrm{H}_{0}: \rho_{i j}=0, \quad 1 \leq i<j \leq p, \\
& \mathrm{H}_{u}: \rho_{i j} \neq 0 .
\end{aligned}
$$

Here $1 \leq i<j \leq p$ denotes the elements in the uppertriangular of the $10 \times 10$ matrix. To be clear, the unconstrained hypothesis is the prior distribution for $\rho_{i j}$. In 
Table 1

Fixed effects parameter estimates

\begin{tabular}{|c|c|c|c|c|c|c|}
\hline \multirow[b]{2}{*}{ Parameter } & \multicolumn{3}{|c|}{ PA Location } & \multicolumn{3}{|c|}{ NA Location } \\
\hline & M & SD & $90 \% \mathrm{CrI}$ & M & $\mathrm{SD}$ & $90 \% \mathrm{CrI}$ \\
\hline$\beta_{0}$ & 0.03 & 0.04 & {$[-0.03,0.10]$} & 0.02 & 0.04 & {$[-0.05,0.09]$} \\
\hline$\beta_{1}$ (Day) $\times 30$ & -0.04 & 0.01 & {$[-0.06,-0.01]$} & -0.03 & 0.01 & {$[-0.05,-0.01]$} \\
\hline$\beta_{2}\left(\mathrm{PA}_{\mathrm{Lag} 1}\right)$ & 0.30 & 0.01 & {$[0.29,0.32]$} & 0.00 & 0.00 & {$[-0.00,0.01]$} \\
\hline$\beta_{3}\left(\mathrm{NA}_{\mathrm{Lag} 1}\right)$ & 0.03 & 0.01 & {$[0.01,0.04]$} & 0.26 & 0.01 & {$[0.24,0.27]$} \\
\hline$\beta_{4}\left(\right.$ Steps $\left._{\mathrm{pm}}\right)$ & 0.15 & 0.07 & {$[0.04,0.25]$} & -0.04 & 0.07 & {$[-0.16,0.06]$} \\
\hline$\beta_{5}\left(\mathrm{Steps}_{\mathrm{pmd}}\right)$ & 0.14 & 0.01 & {$[0.12,0.16]$} & -0.03 & 0.01 & {$[-0.04,-0.02]$} \\
\hline \multirow[t]{2}{*}{$\beta_{6}\left(\right.$ Steps $_{\mathrm{pm}} \times$ Steps $\left._{\mathrm{pmd}}\right)$} & -0.06 & 0.02 & {$[-0.09,-0.02]$} & 0.01 & 0.01 & {$[-0.01,0.02]$} \\
\hline & \multicolumn{3}{|c|}{ PA Scale } & \multicolumn{3}{|c|}{ NA Scale } \\
\hline Parameter & M & SD & $90 \% \mathrm{CrI}$ & M & SD & $90 \% \mathrm{CrI}$ \\
\hline$\eta_{0}$ & -0.81 & 0.04 & {$[-0.87,0.75]$} & -0.90 & 0.04 & {$[-0.97,0.82]$} \\
\hline$\eta_{1}\left(\mathrm{PA}_{\mathrm{Lag} 1}\right)$ & 0.02 & 0.01 & {$[0.00,0.04]$} & 0.05 & 0.01 & {$[\mathbf{0 . 0 3 , 0 . 0 6}]$} \\
\hline$\eta_{2}\left(\mathrm{NA}_{\mathrm{Lag} 1}\right)$ & 0.05 & 0.01 & {$[0.03,0.06]$} & 0.20 & 0.01 & {$[0.18,0.22]$} \\
\hline$\eta_{3}\left(\operatorname{Steps}_{\mathrm{pm}}\right)$ & -0.05 & 0.05 & {$[-0.13,0.04]$} & -0.01 & 0.07 & {$[-0.13,0.10]$} \\
\hline$\eta_{4}\left(\operatorname{Steps}_{\mathrm{pmd}}\right)$ & -0.06 & 0.02 & {$[-0.09,-0.04]$} & -0.08 & 0.03 & {$[-0.13,-0.04]$} \\
\hline$\eta_{5}\left(\operatorname{Steps}_{\mathrm{pm}} \times\right.$ Steps $\left._{\mathrm{pmd}}\right)$ & -0.01 & 0.02 & {$[-0.05,0.03]$} & 0.04 & 0.05 & {$[-0.04,0.12]$} \\
\hline Residual Correlation & -.28 & .01 & {$[-.29,-.26]$} & & & \\
\hline
\end{tabular}

Note: Entries are bolded if the $90 \%$ interval excludes zero, indicating that the sign can be discerned with $\geq 95 \%$ probability.

this case, the hypotheses are nested which allows for using the Savage-Dickey ratio (Dickey, 1971; Wagenmakers, Lodewyckx, Kuriyal, \& Grasman, 2010). Furthermore, the present approach works directly with the correlations $\rho_{i j}$ and the corresponding implied prior distribution derived from the matrix- $F$ prior distribution. Thus, the hypothesis test in favor of the alternative hypothesis can be formulated as

$$
\mathrm{BF}_{10}=\frac{p\left(\mathbf{Y} \mid \mathrm{H}_{1}\right)}{p\left(\mathbf{Y} \mid \mathrm{H}_{0}\right)}=\frac{p\left(\rho_{i j}=0 \mid \mathrm{H}_{1}\right)}{p\left(\rho_{i j}=0 \mid \mathbf{Y}, \mathrm{H}_{1}\right)},
$$

where $\mathrm{H}_{1}$ is the unconstrained hypothesis. In words, by only considering $\mathrm{H}_{1}$ with respect to $\rho_{i j}$, the Bayes factor can be computed as the unconstrained posterior density of $\rho_{i j}$ evaluated at zero divided by the prior density also evaluated at zero (Mulder, Hoijtink, \& de Leeuw, 2012). Importantly, the Bayes factor provides relative evidence between each hypothesis under consideration. In the psychological literature, an analogous approach has been used for both correlations (Marsman \& Wagenmakers, 2017; Wagenmakers, Verhagen, \& Ly, 2016) and partial correlations (Williams \& Mulder, 2019) This requires computing the implied prior for $\rho_{i j}$ from the matrix- $F$ prior that is given in Equation (9). This is represented in Figure 3 (panel $\mathrm{C}$ ), in addition to a hypothetical posterior distribution and the corresponding Bayes factor. In the results section (i.e., Correlations) we follow the customary guidelines provided in Kass and Raftery (1995), wherein a Bayes factor greater than 3 is considered positive (relative) evidence for a given hypothesis.

\section{Estimation and Software}

The fitted model included four chains of 1,000 iterations each, excluding a warm-up period of the same size. This number of iterations provided a good quality of the parameter estimates in which the models converged with potential scale reduction factors $\hat{R}$ smaller than 1.1 (Gelman, 2006). We summarize each posterior distribution with the mean, standard deviation, and a 90\% equal-tailed credible interval $(\mathrm{CrI})$. We also report directional posterior probabilities greater than or less than zero in the text-i.e., $p(\theta>0 \mid \mathbf{Y}, \mathcal{M})$. This provides context to findings, for example to compare the magnitude of the respective effects between constructs. In a Bayesian framework these differences can be computed by simply subtracting the posterior distributions (Table 1 and 2).

All computations were done in $\mathrm{R}$ version 3.5.2 ( $\mathrm{R}$ Core Team, 2017). The model was fitted with the the package brms (Bürkner, 2017b), which serves as a front-end to the probabilistic programming language Stan (Stan Development Team, 2016). There are several advantages of the package brms. The model specification follows that of lme4 (Bates, Mächler, Bolker, \& Walker, 2015), although brms allows for fitting a much wider range of models. Additionally, 
there are several post-processing features for model checking and Bayesian hypothesis testing. There are also several tutorials describing brms, including for ordinal models (Burkner \& Vuorre, 2017), distributional regression (link to html), and supporting code for an introductory Bayesian textbook on mixed models (link to repo).

\section{Results}

\section{Fixed Effects}

Location. The fixed effects are reported in Table 1. The estimate of day was small and negative for both constructs, thus indicating a decrease in PA and NA over time. For PA both lagged predictors increased the reported rating, with a $100 \%$ posterior probability, on the following day. This was not the case for NA. Not only was $\beta_{2}^{(\mathrm{NA})}\left(\mathrm{PA}_{\mathrm{Lag} 1}\right)$ smaller than $\beta_{3}^{(\mathrm{PA})}\left(\mathrm{NA}_{\mathrm{Lag} 1}\right)$, with a $100 \%$ probability, but the former had (only) a $84 \%$ probability of increasing the rating of NA. For physical activity more steps, on average, was associated with higher levels of PA, whereas this was not observed for NA. Note that $90 \% \mathrm{CrI}$ for the difference included zero, which indicates the effect was not "significantly" different between constructs. On the other hand, the predictor Steps pmd captured within-person differences from their daily average step count. Here, deviating positively from the average step count increased ratings of PA and decreased ratings of NA. The interaction for positive affect is displayed in Figure 1 (panel D). This reveals that individuals, who on average walked less than others, were also most responsive to deviating from their mean step count. In other words, for a relatively inactive person, walking more than their typical day was associated with higher ratings of PA, whereas active individuals were apparently less responsive to walking more on a given day.

Scale. The scale (i.e., intraindividual variability) fixed effects are also provided in Table 1. The lagged effects point towards some interesting findings, in that the previous days rating of PA and NA influenced fluctuations in the respective trait. That is, there was a positive relationship between within-person variance and yesterdays affective rating. However, the direction of these effects was perhaps counter intuitive: For each lagged effect, there was an increase in withinperson variance. Note also that the largest lagged effect was $\eta_{1}^{\mathrm{NA}}\left(\mathrm{NA}_{\text {Lag } 1}\right)$, wherein the others were smaller in magnitude with a $100 \%$ posterior probability. Furthermore, there was also a relation between each outcome and deviating from an individuals mean step count $\eta_{4}\left(\mathrm{Steps}_{\mathrm{pmd}}\right)$. That is, if someone walked more than their respective average, this was associated with more stability in that trait.

\section{Random Effects}

For the random effects we depart from customary approaches that focus on detecting non-zero variance components and instead take a more descriptive approach. They are plotted in Figure 2 and centered at the fixed effect value. The blue intervals correspond to $90 \%$ CrI's that exclude the respective fixed effect. The corresponding standard deviations are provided in Table 2.

The figure reveals individual variation for each parameter, in that all had several people who differed from the fixed effect. The dotted line at zero can also be used as a reference point, for example to indicate how many individuals had a "significant" effect and/or in what direction. While there was an effect of day, for both constructs, it was not so simple as ratings decreasing over time (e.g., participants experiencing study fatigue). For positive affect in particular, $24 \%$ of the sample decreased in their rating, whereas $13 \%$ showed an increase in PA over time (an effect in the opposite direction). The effect of $\beta_{5}\left(\mathrm{Step}_{\mathrm{pmd}}\right)$ was more consistent across the sample. Here only one person showed an effect in the opposite direction, wherein deviating from their respective (average) step count reduced feelings of PA. Further, almost half of the sample showed a positive effect. Said another way, when a person took more steps than on their average day, this was associated with higher ratings of PA in 1 out of 2 individuals. A similar pattern was observed for NA.

We now discuss the scale random effects. Here the intercepts correspond to within-person variance at the beginning of the study. In reference to the fixed effect, $\eta_{0}$, there was considerable individual variation. That is, for both constructs, $\approx 75 \%$ of the individual effects differed from the fixed effect. The fixed effect of $\eta_{4}\left(\right.$ Step $\left._{\mathrm{pmd}}\right)$ indicated that taking more steps, than average, was related to stability in each construct. Importantly, this deserves some nuance because, in fact, there was an effect in the opposite direction for 6 (PA) and 15 (NA) participants. In other words, some people became more variable in their emotional states when they walked more than on average. These inferences are made possible by inspecting the individual (random) effects, and we encourage applied researcher to similarly go beyond significance testing of variance components (which was not pursued here).

It is straightforward to compare the posterior distributions-i.e., $s d\left(u_{0 i}^{(\mathrm{PA})}\right)-s d\left(u_{0 i}^{(\mathrm{NA})}\right)$. This allows for asking whether the random effects, for a particular construct, were more variable than the other (Table 2). When comparing the standard deviations of $\beta_{5}\left(\mathrm{Step}_{\mathrm{pmd}}\right), s d\left(u_{1 i}\right)$, this revealed that the random effects of NA were more variable than for PA (100\% posterior probability). That is, individuals were more widely dispersed around the fixed effect estimate. A similar pattern was observed for the scale random effects standard deviations. Interestingly, at the beginning of the study, $s d\left(u_{3 i}\right)$, there was more variability in the within-person variance for NA. Here the probability was again $100 \%$. This pattern also extended to $\eta_{4} \mathrm{Step}_{\mathrm{pmd}}$, where the random effects were again more variable for NA. Together, this points towards more individual variation in 

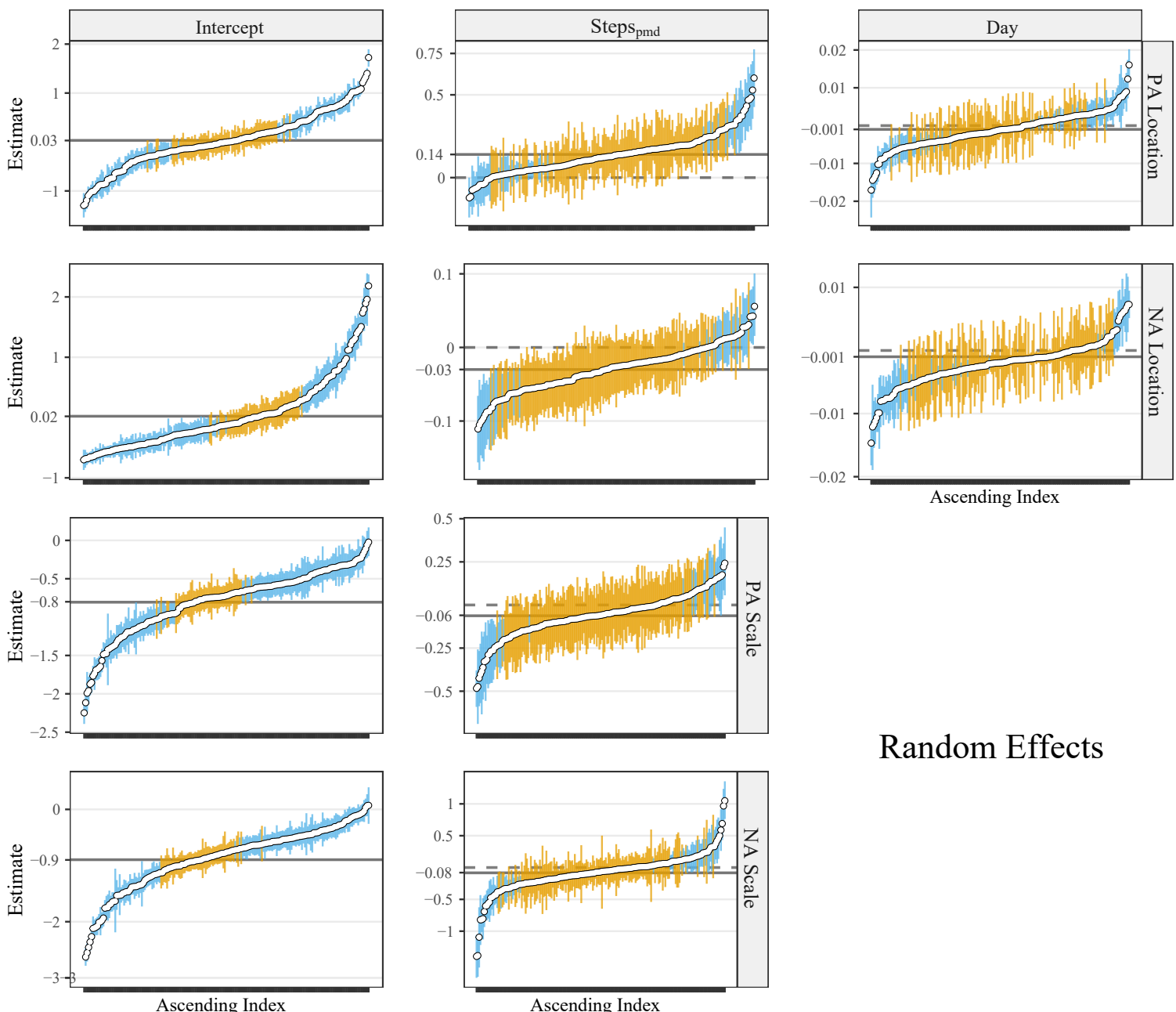

\section{Random Effects}

Figure 2. Subject-specific posterior estimates and $90 \%$ intervals (in ascending order) for the intercepts and coefficients of the location and scale of both PA and NA. The solid line is the fixed effect, and the dashed line is zero. Intervals are blue if they exclude the fixed effect, and orange otherwise. Note that many individuals (blue) are inadequately described by the average effect, and several have effects in the opposite direction (in reference to the dashed lines).

NA (the trait), in relation to physical activity, and also in the overall stability (or conversely instability) of the states.

\section{Correlations}

An additional advantage of the proposed model is that correlations between location and scale random effects can be estimated, as well as between constructs (due to the multivariate formulation). The full correlation matrix is provided in Table 3. The Bayes factors were computed on the logarithmic scale and are reported in the upper-triangular.

Location ( $\mu$ parameters). In the following we discuss some noteworthy correlations. For example, there was evidence for a negative correlation between both constructs in relation to deviating from their average daily step count (correlation among $u_{2}^{(P A)}$ and $u_{2}^{(N A)}$ ). Walking more than average was associated to higher PA and lower NA $(r=-0.60$,
$\left.\mathrm{BF}_{10}=6.63\right)$. There was a positive relation between PA on day 1 (i.e., $u_{0}^{(P A)}$ the intercept) and $\operatorname{Steps}_{\mathrm{pmd}}^{(\mathrm{NA})}\left(u_{2}^{(N A)}\right)$, such that those with higher trait PA were less responsive to the effect physical activity on ratings of NA $\left(r=0.30, \mathrm{BF}_{10}=1.62\right)$. On the other hand, when considering NA at day $1\left(u_{0}^{(N A)}\right)$ and $\mathrm{Step}_{\mathrm{pmd}}^{(\mathrm{PA})}\left(u_{2}^{(P A)}\right)$, there was evidence for the null hypothesis $\left(r=-0.07, \mathrm{BF}_{10}=-1.47\right)-$ i.e., relative to the unconstrained hypothesis (Figure 3; panel C), trait NA was not related to physical activity and PA.

Scale ( $\sigma^{2}$ parameters). There was a positive relation between constructs for within-person variance $\left(u_{3}^{(P A)}\right.$ and $\left.u_{3}^{(N A)}\right)$ at the beginning of the study $\left(r=0.58, \mathrm{BF}_{10}=35.15\right)$. In other words, individuals that fluctuated around their mean for one trait tended to also fluctuate around the mean of the other trait. Further, as described above, the fixed effect $\eta_{4}\left(\right.$ Steps $\left._{\text {pmd }}\right)$ indicated that taking more steps, than aver- 
Table 2

Random effects parameter estimates

\begin{tabular}{|c|c|c|c|c|c|c|}
\hline \multirow[b]{2}{*}{ Parameter } & \multicolumn{3}{|c|}{ PA Location } & \multicolumn{3}{|c|}{ NA Location } \\
\hline & M & $\mathrm{SD}$ & $90 \% \mathrm{CrI}$ & M & $\mathrm{SD}$ & $90 \% \mathrm{CrI}$ \\
\hline Intercept $s d\left(u_{0 i}^{k}\right)$ & 0.59 & 0.03 & {$[0.54,0.65]$} & 0.61 & 0.03 & {$[0.57,0.69]$} \\
\hline Day $s d\left(u_{1 i}^{k}\right)$ & 0.01 & 0.00 & {$[0.01,0.01]$} & 0.00 & 0.00 & {$[0.00,0.01]$} \\
\hline \multirow[t]{2}{*}{ Steps $_{\text {pmd }} s d\left(u_{2 i}^{k}\right)$} & 0.16 & 0.01 & {$[0.13,0.18]$} & 0.35 & 0.03 & {$[0.31,0.40]$} \\
\hline & \multicolumn{3}{|c|}{ PA Scale } & \multicolumn{3}{|c|}{ NA Scale } \\
\hline Parameter & M & $\mathrm{SD}$ & $90 \% \mathrm{CrI}$ & M & $\mathrm{SD}$ & $90 \% \mathrm{CrI}$ \\
\hline Intercept $s d\left(u_{3 i}^{k}\right)$ & 0.45 & 0.03 & {$[0.41,0.49]$} & 0.63 & 0.03 & {$[0.57,0.69]$} \\
\hline Steps $_{\text {pmd }} s d\left(u_{4 i}^{k}\right)$ & 0.17 & 0.02 & {$[0.14,0.20]$} & 0.35 & 0.03 & {$[0.31,0.40]$} \\
\hline
\end{tabular}

Table 3

Random effects correlations

\begin{tabular}{|c|c|c|c|c|c|c|c|c|c|c|c|}
\hline & & \multicolumn{3}{|c|}{$\mu$} & \multicolumn{2}{|c|}{$\sigma^{2}$} & \multicolumn{3}{|c|}{$\mu$} & \multicolumn{2}{|c|}{$\sigma^{2}$} \\
\hline & & $u_{0 i}^{(\mathrm{PA})}$ & $u_{1 i}^{(\mathrm{PA})}$ & $u_{2 i}^{(\mathrm{PA})}$ & $u_{3 i}^{(\mathrm{PA})}$ & $u_{4 i}^{(\mathrm{PA})}$ & $u_{0 i}^{(\mathrm{NA})}$ & $u_{1 i}^{(\mathrm{NA})}$ & $u_{2 i}^{(\mathrm{NA})}$ & $u_{3 i}^{(\mathrm{NA})}$ & $u_{4 i}^{(\mathrm{NA})}$ \\
\hline \multirow{3}{*}{$\mu$} & $u_{0 i}^{(\mathrm{PA})}$ & & 2.74 & -0.81 & 7.77 & 3.07 & 1.09 & -0.28 & 1.62 & 9.60 & 0.60 \\
\hline & $u_{1 i}^{(\mathrm{PA})}$ & $-0.24^{\dagger}$ & & -1.20 & -1.83 & -1.21 & 0.65 & 1.41 & -1.16 & -1.10 & -0.43 \\
\hline & $u_{2 i}^{(\mathrm{PA})}$ & -0.13 & $-0.10^{\ddagger}$ & & 3.17 & 2.13 & -1.47 & -0.93 & 6.63 & -0.92 & 0.49 \\
\hline \multirow{3}{*}{$\sigma^{2}$} & $u_{3 i}^{(\mathrm{PA})}$ & $-0.33^{\dagger}$ & -0.04 & $0.26^{\dagger}$ & & -1.56 & -0.26 & -1.72 & -0.58 & 35.15 & -1.42 \\
\hline & $u_{4 i}^{(\mathrm{PA})}$ & $-0.30^{\dagger}$ & $-0.10^{\ddagger}$ & $-0.34^{\dagger}$ & $0.05^{\ddagger}$ & & 1.58 & -1.49 & -1.21 & 1.34 & 4.67 \\
\hline & $u_{0 i}^{(\mathrm{NA})}$ & -0.18 & 0.19 & $-0.07^{\ddagger}$ & 0.14 & $0.26^{\dagger}$ & & 4.58 & 0.15 & 20.92 & -1.45 \\
\hline \multirow[t]{2}{*}{$\mu$} & $u_{1 i}^{(\mathrm{NA})}$ & 0.15 & $-0.26^{\dagger}$ & 0.13 & $0.05^{\ddagger}$ & $-0.05^{\ddagger}$ & $-0.35^{\dagger}$ & & -0.93 & -1.79 & -1.69 \\
\hline & $u_{2 i}^{(\mathrm{NA})}$ & $0.30^{\dagger}$ & $0.04^{\ddagger}$ & $-0.60^{\dagger}$ & -0.16 & $0.07^{+}$ & -0.29 & -0.07 & & 1.25 & 9.01 \\
\hline \multirow{2}{*}{$\sigma^{2}$} & $u_{3 i}^{(\mathrm{NA})}$ & $-0.35^{\dagger}$ & $0.10^{\ddagger}$ & 0.12 & $0.58^{\dagger}$ & $0.24^{\dagger}$ & $0.47^{\dagger}$ & $-0.04^{\ddagger}$ & $-0.25^{\dagger}$ & & -1.95 \\
\hline & $u_{4 i}^{(\mathrm{NA})}$ & -0.18 & 0.15 & -0.20 & $0.08^{\ddagger}$ & $0.38^{\dagger}$ & $0.08^{\ddagger}$ & $-0.04^{\ddagger}$ & $0.55^{\dagger}$ & $0.01^{\ddagger}$ & \\
\hline
\end{tabular}

Note: $\quad{ }^{\dagger} H_{1}$ is supported. ${ }^{\ddagger} H_{0}$ is supported.

Correlation estimates are provided below the diagonal, with corresponding $\log \mathrm{BF}_{10}$ values above the diagonal.

age, reduced within-person variance for both constructs (Table 1). Accordingly, the random effects correlation between constructs was positive $\left(r=0.38, \mathrm{BF}_{10}=4.67\right)$. This suggests that, when walking more than on a typical day, individuals who became more stable for PA also became more stable for NA. Note that this inference is made possible by the multivariate formulation (Equation 4), in addition to estimating the full covariance structure (Section Random Effects Variance Model). When considering IIV at day 1 for $\mathrm{PA}$, in relation to $\eta_{4}\left(\mathrm{Steps}_{\mathrm{pmd}}\right)$ predicting both PA $(r=0.05$, $\left.\mathrm{BF}_{10}=-1.56\right)$ and NA $\left(r=0.08, \mathrm{BF}_{10}=-1.42\right)$, there was evidence for the null hypothesis. In other words, more variable individuals were not more (or less) responsive to the dampening effect of physical activity on positive affect.
Location and Scale. A special feature of the MELSM are random effects correlations across the location and the scale. There were negative relations between the location of $\mathrm{PA}$, at the beginning of the study, and within-person variance in both constructs. In other word, those who reported higher PA to begin with were also more stable in PA $(r=-0.33$ $\left.\mathrm{BF}_{10}=7.78\right)$ and NA $\left(r=-0.35 \mathrm{BF}_{10}=9.60\right)$. Conversely, the opposite was observed for NA, such that higher ratings of $\mathrm{NA}$ at day 1 were associated with more within-person variance in NA $\left(r=0.46 \mathrm{BF}_{10}=20.92\right)$. Interestingly, those who had a larger effect for physical activity on ratings of PA were also more responsive to physical activity as it related to reducing fluctuations in $\mathrm{PA}\left(r=-0.34 \mathrm{BF}_{10}=2.14\right)$. 


\section{Discussion}

In this paper we extended the standard mixed-effects location scale model, by fitting two outcomes simultaneously, and allowing for individual variation therein. This model was conceptualized to address the goal of identifying and accounting for IIV in personality traits. Intensive longitudinal modeling, with the goal of explaining traits at both the mean and variance level, requires repeated trials and flexible methods that are able to capture changes within and differences between individuals, of which the M-MELSM is one such model. This approach can simultaneously model both the personality traits (the mean structure) and states (the variance structure) for multivariate data common to personality research. Moreover, variables can be included to predict either (or both) the traits and states simultaneously. The model also provides random effects for both the location and the scale components, thus allowing for characterizing the uncertainty of individual effects. As a result, there are correlations between location and scale random effects, both across and within each outcome, which allows characterizing the interplay between personality traits and states.

\section{Substantive Applications}

In addition to affect, there are many applications where the M-MELSM may be fruitfully applied in personality research. For example, the model formulation seamlessly generalizes to any number of outcomes. This is ideal for personality assessment, in that traits can be modeled simultaneously (perhaps the big 5 inventory). In this case, we anticipate the model will need fully informed priors for each parameter to ensure convergence. Note that prior distributions, in addition to serving as hypotheses, can also be used to constrain the parameter space (Gelman, Simpson, \& Betancourt, 2017). This can improve the quality of estimates, reduce computation time, and allows for estimating complex models. Thus, the presented model provides a flexible approach that allows for asking novel research questions to elucidate both inter and intraindividual variability in personality traits.

Bayesian estimation has become more accessible and popular over the last years as it entered mainstream software packages. In fact, the models described here were fit with the $\mathrm{R}$ package brms which uses similar syntax as lme4 (Bürkner, 2017a). In our experience, brms is sufficiently flexible to fit most models in psychology, but Stan can be used directly if needed. For example, it is possible for another sub-model predicting the between-person variance (Rast \& Ferrer, 2018). While Bayesian estimation techniques have become more widespread, the same can not be said about Bayesian inference. While a thorough discussion on that topic (e.g., on hypothesis testing) was beyond the scope of this work we illustrated some of the possibilities that Bayesian inference is able to offer. There are now several introductions for Bayesian inference specifically for psychological applications (Quintana \& Williams, 2018). These are typically geared towards simpler models (e.g., $t$ test; Rouder, Speckman, Sun, Morey, \& Iverson, 2009), but the techniques can be used with the M-MELSM. We refer to Wagenmakers, Marsman, et al. (2018) and Wagenmakers, Love, et al. (2018), in addition to Wagenmakers et al. (2010) which is specifically about the Savage-Dickey ratio. Importantly, Bayes factors depend critically upon the prior distribution, which should ideally be informed by relevant theory. In the absence of precise theoretical predictions, it is common place to assume defaults (Rouder \& Morey, 2012). In practice, when there is prior uncertainty, sensitivity analyses should be performed. They were not included in this work for simplicity. We refer to Carlsson, Schimmack, Williams, and Bürkner (2017), where the prior distributions were varied as a robustness check.

\section{Limitations}

There are notable limitations of this work. When the variances are of interest, it should be noted that their magnitude is also defined by the location of the average response. In other words, with bounded variables that are common in psychology, the variance will be a function of the person's mean (Baird, Le, \& Lucas, 2006; Eid \& Diener, 1999; Kalmijn \& Veenhoven, 2005; Rouder, Tuerlinckx, Speckman, Lu, \& Gomez, 2008). This problem is known in MELSM applications (Rast \& Ferrer, 2018), but also applies to the M-MELSM. These effects could be of substantive interest, or dictated by aspects of the study design. This should be considered when making inference from the random effect correlations. Moreover, because our goal was to introduce a Bayesian M-MELSM to personality assessment, many choices were made for simplicity. For example, we did not provide an in-depth example of model checking, but note this is important in practical applications (see Gabry, Simpson, Vehtari, Betancourt, \& Gelman, 2019). Further, we discussed only those fixed effects in which the CrI excluded zero, but evidence for the null hypothesis was not evaluated. In practical applications this would be possible by defining an informative prior and computing a Bayes factor or by defining a region of practical equivalence (ROPE; Kruschke, 2011). Importantly, for those effects not discussed, the reported estimates (Table 1 and 2) can be used to infer which values are included in the CrI (in addition to zero). Lastly, we assumed improper prior distributions for the fixed effects. This decision was again made to keep the model formulation concise, although in practice we would use (at minimum) weakly informative prior distributions (see Gelman, 2006; Gelman, Jakulin, Pittau, \& Su, 2008; Williams, Rast, \& Bürkner, 2018). 


\section{Conclusion}

The purpose of this paper was to present the M-MELSM as a flexible tool for personality assessment. Our proposed model is suited for the "Big 2" of affect, as presented in this work, but can also be used more generally in personality research (e.g., the Big 5). By focusing on the within-person variance, this approach opened up possibilities for modeling a component that is often disregarded as "noise". The application highlighted such possibilities and demonstrated that the residual variance may show systematic patterns that are important for understanding the interplay between personality traits and states.

\section{References}

Augustine, A. A., \& Larsen, R. J. (2015). Personality, affect, and affect regulation. In Apa handbook of personality and social psychology, volume 4: Personality processes and individual differences. (pp. 147-165). Washington, DC, US: American Psychological Association. doi: 10.1037/14343-007

Baird, B. M., Le, K., \& Lucas, R. E. (2006). On the nature of intraindividual personality variability: Reliability, validity, and associations with well-being. Journal of personality and social psychology, 90(3), 512.

Bates, D., Mächler, M., Bolker, B., \& Walker, S. (2015). Fitting Linear Mixed-Effects Models Using \{lme4\}. Journal of Statistical Software, 67(1), 1-48. doi: 10.18637/jss.v067.i01

Burger, J. M., \& Caldwell, D. F. (2000). Personality, Social Activities, Job-Search Behavior and Interview Success: Distinguishing between PANAS Trait Positive Affect and NEO Extraversion. Motivation and Emotion, 24(1), 51-62. doi: 10.1023/A:1005539609679

Bürkner, P.-C. (2017a). Advanced Bayesian Multilevel Modeling with the R Package brms. , 1-18. Retrieved from http://arxiv.org/abs/1705.11123

Bürkner, P.-C. (2017b). brms : An R Package for Bayesian Multilevel Models Using Stan. Journal of Statistical Software, 80(1). doi: 10.18637/jss.v080.i01

Burkner, P.-C., \& Vuorre, M. (2017). Ordinal Models in Psychology : A Tutorial., 1-17.

Carlsson, R., Schimmack, U., Williams, D. R., \& Bürkner, P. C. (2017). Bayes Factors From Pooled Data Are No Substitute for Bayesian Meta-Analysis: Commentary on Scheibehenne, Jamil, and Wagenmakers (2016) (Vol. 28) (No. 11). doi: $10.1177 / 0956797616684682$

Cattell, R. B., Cattell, A. K. S., \& Rhymer, R. M. (1947). Ptechnique demonstrated in determining psychophysiological source traits in a normal individual. Psychometrika, 12(4), 267-288.

Chida, Y., \& Hamer, M. (2008). Chronic Psychosocial Factors and Acute Physiological Responses to Laboratory-Induced Stress in Healthy Populations: A Quantitative Review of 30 Years of Investigations. Psychological Bulletin, 134(6), 829885. doi: $10.1037 / \mathrm{a} 0013342$

Cobb-Clark, D. A., \& Schurer, S. $(2012,4)$. The stability of bigfive personality traits. Economics Letters, 115(1), 11-15. doi: 10.1016/j.econlet.2011.11.015
Cohen, S., \& Pressman, S. (2005). Positive Affect and Health. Current Directions in Psychological Science, 15(3), 122-125.

Costa, P. T., \& McCrae, R. R. (1988, 5). Personality in adulthood: a six-year longitudinal study of self-reports and spouse ratings on the NEO Personality Inventory. Journal of personality and social psychology, 54(5), 853-63.

Dickey, J. M. (1971, 2). The Weighted Likelihood Ratio, Linear Hypotheses on Normal Location Parameters. The Annals of Mathematical Statistics, 42(1), 204-223. doi: 10.1214/ aoms/1177693507

Eid, M., \& Diener, E. (1999). Intraindividual variability in affect: Reliability, validity, and personality correlates. Journal of Personality and Social Psychology, 76(4), 662.

Estabrook, R., Grimm, K. J., \& Bowles, R. P. (2012, 9). A Monte Carlo simulation study of the reliability of intraindividual variability. Psychology and Aging, 27(3), 560-576. doi: 10.1037/a0026669

Finch, J. F., Baranik, L. E., Liu, Y., \& West, S. G. (2012). Physical health, positive and negative affect, and personality: A longitudinal analysis. Journal of Research in Personality, 46(5), 537-545. doi: 10.1016/j.jrp.2012.05.013

Fiske, D. W., \& Rice, L. (1955). Intra-individual response variability. Psychological Bulletin, 52(3), 217.

Fleeson, W. (2001). Toward a structure-and process-integrated view of personality: Traits as density distributions of states. Journal of personality and social psychology, 80(6), 1011-27.

Fleeson, W., \& Law, M. K. $(2015,12)$. Trait enactments as density distributions: The role of actors, situations, and observers in explaining stability and variability. Journal of Personality and Social Psychology, 109(6), 1090-1104. doi: 10.1037/a0039517

Gabry, J., Simpson, D., Vehtari, A., Betancourt, M., \& Gelman, A. (2019). Visualization in Bayesian workflow. J.R. Statist. Soc. A(1), 1-14.

Gelman, A. (2006). Prior distribution for variance parameters in hierarchical models. Bayesian Analysis, 1(3), 515-533. doi: 10.1214/06-BA117A

Gelman, A., Jakulin, A., Pittau, M. G., \& Su, Y.-S. (2008). A Weakly Informative Default Prior Distribution for Logistic and Other Regression Models. The Annals of Applied Statistics, 2(4), 1360-1383. doi: 10.1214/08-AOAS

Gelman, A., Simpson, D., \& Betancourt, M. (2017). The prior can generally only be understood in the context of the likelihood. ArXiv preprint. Retrieved from http://arxiv.org/abs/ 1708.07487

Goldstein, H. (2011). Multilevel statistical models (Vol. 922). John Wiley \& Sons.

Hamaker, E. L., \& Grasman, R. P. P. P. (2015, 1). To center or not to center? Investigating inertia with a multilevel autoregressive model. Frontiers in Psychology, 5, 1492. doi: 10.3389/fpsyg.2014.01492

Hardy, J., \& Segerstrom, S. C. (2017). Intra-individual variability and psychological flexibility: Affect and health in a National US sample. Journal of Research in Personality, 69, 13-21. doi: 10.1016/j.jrp.2016.04.002

Hedeker, D., Mermelstein, R. J., \& Demirtas, H. (2012). Modeling between-subject and within-subject variances in ecological momentary assessment data using mixed-effects location 
scale models. Statistics in Medicine, 31(27), 3328-3336. doi: $10.1002 / \operatorname{sim} .5338$

Hedeker, D., \& Nordgren, R. (2013). MIXREGLS : A Program for Mixed-Effects Location. Journal of Statistical Software, 52(12), 1-38.

Hepburn, L., \& Eysenck, M. W. (1989, 1). Personality, average mood and mood variability. Personality and Individual Differences, 10(9), 975-983. doi: 10.1016/0191-8869(89) 90062-7

Horn, J. L. (1972). State, trait and change dimensions of intelligence. British Journal of Educational Psychology, 42(2), 159-185.

Hutteman, R., Back, M. D., Geukes, K., Küfner, A. C., \& Nestler, S. (2016). Trait personality and state variability: Predicting individual differences in within- and cross-context fluctuations in affect, self-evaluations, and behavior in everyday life. Journal of Research in Personality, 69, 124-138. doi: 10.1016/j.jrp.2016.06.003

Kalin, A. M., Plfuger, M., Gietl, A. F., Riese, F., JÃncke, L., Nitsch, R. M., \& Hock, C. (2014, 7). Intraindividual variability across cognitive tasks as a potential marker for prodromal Alzheimers disease. Frontiers in Aging Neuroscience, 6, 147. doi: 10.3389/fnagi.2014.00147

Kalmijn, W., \& Veenhoven, R. (2005). Measuring inequality of happiness in nations: In search for proper statistics. Journal of Happiness Studies, 6(4), 357-396.

Kapur, K., Li, X., Blood, E. A., \& Hedeker, D. (2015, 2). Bayesian mixed-effects location and scale models for multivariate longitudinal outcomes: an application to ecological momentary assessment data. Statistics in Medicine, 34(4), 630-651. doi: 10.1002/sim.6345

Kass, R. E., \& Raftery, A. E. (1995). Bayes Factors. Journal of the American Statistical Association, 90(430), 773-795.

Kiecolt-Glaser, J. K., McGuire, L., Robles, T. F., \& Glaser, R. (2002). Psychoneuroimmunology: Psychological influences on immune function and health. Journal of Consulting and Clinical Psychology, 70(3), 537-547. doi: 10.1037/ 0022-006X.70.3.537

Kruschke, J. K. (2011). Bayesian Assessment of Null Values Via Parameter Estimation and Model Comparison. Perspectives on Psychological Science, 6, 299-312. doi: 10.1177/ 1745691611406925

Kubzansky, L. D., \& Kawachi, I. (2000). Going to the heart of the matter: Do negative emotions cause coronary heart disease? Journal of Psychosomatic Research, 48(4-5), 323-337. doi: 10.1016/S0022-3999(99)00091-4

Kuppens, P., Van Mechelen, I., Nezlek, J. B., Dossche, D., \& Timmermans, T. (2007). Individual differences in core affect variability and their relationship to personality and psychological adjustment. Emotion, 7(2), 262-274. doi: 10.1037/1528-3542.7.2.262

Leckie, G., French, R., Charlton, C., \& Browne, W. (2014). Modeling heterogeneous variance-covariance components in twolevel models. Journal of Educational and Behavioral Statistics, 39(5), 307-332.

Lewandowski, D., Kurowicka, D., \& Joe, H. (2009). Generating random correlation matrices based on vines and extended onion method. Journal of Multivariate Analysis, 100(9),
1989-2001. doi: 10.1016/j.jmva.2009.04.008

Lucas, R. E., Le, K., \& Dyrenforth, P. S. (2008, 6). Explaining the Extraversion/Positive Affect Relation: Sociability Cannot Account for Extraverts' Greater Happiness. Journal of Personality, 76(3), 385-414. doi: 10.1111/j.1467-6494.2008 $.00490 . \mathrm{x}$

Maccallum, R., Kim, C., William, B., \& Kiecolt-Glaser, J. (1997). Multivariate behavioral studying multivariate change using multilevel models and latent curve models. Multivariate Behavioral Research, 32(August 2013), 215-253. doi: 10 $.1207 / \mathrm{s} 15327906 \mathrm{mbr} 3203$

MacDonald, S. W. S., Hultsch, D. F., \& Dixon, R. A. (2008). Predicting impending death: inconsistency in speed is a selective and early marker. Psychology and aging, 23(3), 595.

Marsman, M., \& Wagenmakers, E. J. (2017). Bayesian benefits with JASP. European Journal of Developmental Psychology, 14(5), 545-555. doi: 10.1080/17405629.2016.1259614

Martin, P., Long, M. V., \& Poon, L. W. (2002, 3). Age changes and differences in personality traits and states of the old and very old. Journals of Gerontology - Series B Psychological Sciences and Social Sciences, 57(2), P144-P152. doi: 10.1093/geronb/57.2.P144

Mestdagh, M., Pe, M., Pestman, W., Verdonck, S., Kuppens, P., \& Tuerlinckx, F. (2018, 12). Sidelining the mean: The relative variability index as a generic mean-corrected variability measure for bounded variables. Psychological Methods, 23(4), 690-707. doi: 10.1037/met0000153

Mulder, J., Hoijtink, H., \& de Leeuw, C. (2012). BIEMS: A Fortran 90 program for calculating $\{\mathrm{B}\}$ ayes factors for inequality and equality constrained model. Journal of Statistical Software, 46.

Mulder, J., \& Raúl Pericchi, L. (2018). The Matrix-F Prior for Estimating and Testing Covariance Matrices. Bayesian Analysis(4), 1-22. doi: 10.1214/17-BA1092

Paluska, S. A., \& Schwenk, T. L. (2000). Physical Activity and Mental Health. Sports Medicine, 29(3), 167-180. doi: 10.2165/00007256-200029030-00003

Quintana, D. S., \& Williams, D. R. (2018). Bayesian alternatives for common null-hypothesis significance tests in psychiatry: A non-technical guide using JASP. BMC Psychiatry, 18(1). doi: 10.1186/s12888-018-1761-4

R Core Team. (2017). R: A Language and Environment for Statistical Computing. Vienna, Austria: R Foundation for Statistical Computing. Retrieved from https://www.r-project .org/

Ram, N., \& Gerstorf, D. (2009). Time-structured and net intraindividual variability: Tools for examining the development of dynamic characteristics and processes. Psychology and aging, 24(4), 778.

Rast, P., \& Ferrer, E. (2018). A Mixed-Effects Location Scale Model for Dyadic Interactions. , 1-63. doi: 10.1080/ 00273171.2018.1477577

Rast, P., Hofer, S. M., \& Sparks, C. (2012). Modeling Individual Differences in Within-Person Variation of Negative and Positive Affect in a Mixed Effects Location Scale Model Using BUGS/JAGS. Multivariate Behavioral Research, 47(2), 177-200. doi: 10.1080/00273171.2012.658328

Röcke, C., Li, S.-C., \& Smith, J. (2009, 12). Intraindividual vari- 
ability in positive and negative affect over 45 days: Do older adults fluctuate less than young adults? Psychology and Aging, 24(4), 863-878. doi: 10.1037/a0016276

Rouder, J. N., \& Morey, R. D. (2012). Default Bayes Factors for Model Selection in Regression. Multivariate Behavioral Research, 47(6), 877-903. doi: 10.1080/00273171.2012 .734737

Rouder, J. N., Speckman, P. L., Sun, D., Morey, R. D., \& Iverson, G. (2009). Bayesian $t$ tests for accepting and rejecting the null hypothesis. Psychonomic Bulletin and Review, 16(2), 225-237. doi: 10.3758/PBR.16.2.225

Rouder, J. N., Tuerlinckx, F., Speckman, P., Lu, J., \& Gomez, P. (2008). A hierarchical approach for fitting curves to response time measurements. Psychonomic Bulletin and Review, 15(6), 1201-1208. doi: 10.3758/PBR.15.6.1201

Salthouse, T. A. (2007). Implications of within-person variability in cognitive and neuropsychological functioning for the interpretation of change. Neuropsychology, 21(4), 401.

Sliwinski, M. J., Almeida, D. M., Smyth, J., \& Stawski, R. S. (2009). Intraindividual change and variability in daily stress processes: Findings from two measurement-burst diary studies. Psychology and Aging, 24(4), 828.

Stan Development Team. (2016). Rstan: the R interface to Stan. Retrieved from http://mc-stan.org/

Suls, J., \& Bunde, J. (2005). Anger, anxiety, and depression as risk factors for cardiovascular disease: The problems and implications of overlapping affective dispositions. Psychological Bulletin, 131(2), 260-300. doi: 10.1037/0033-2909.131.2 .260

Timmermans, T., Van Mechelen, I., \& Kuppens, P. (2010, 12). The relationship between individual differences in intraindividual variability in core affect and interpersonal behaviour. European Journal of Personality, 24(8), 623-638. doi: 10.1002/ per.756

Vazire, S., \& Sherman, R. A. (2017). Introduction to the special issue on within-person variability in personality. Journal of Research in Personality, 69, 1-3. doi: 10.1016/ j.jrp.2017.07.004

Wagenmakers, E. J., Lodewyckx, T., Kuriyal, H., \& Grasman, R. (2010). Bayesian hypothesis testing for psychologists: A tutorial on the Savage-Dickey method. Cognitive Psychology, 60(3), 158-189. doi: 10.1016/j.cogpsych.2009.12.001

Wagenmakers, E. J., Love, J., Marsman, M., Jamil, T., Ly, A., Verhagen, J., ... Morey, R. D. (2018). Bayesian inference for psychology. Part II: Example applications with JASP. Psychonomic Bulletin and Review, 25(1), 58-76. doi: 10.3758/ s13423-017-1323-7

Wagenmakers, E. J., Marsman, M., Jamil, T., Ly, A., Verhagen, J., Love, J., ... Morey, R. D. (2018). Bayesian inference for psychology. Part I: Theoretical advantages and practical ramifications. Psychonomic Bulletin and Review, 25(1), 3557. doi: 10.3758/s13423-017-1343-3

Wagenmakers, E.-J., Verhagen, J., \& Ly, A. (2016). How to quan- tify the evidence for the absence of a correlation. Behavior Research Methods, 48(2), 413-426.

Wang, L. P., \& Grimm, K. J. (2012, 9). Investigating Reliabilities of Intraindividual Variability Indicators. Multivariate Behavioral Research, 47(5), 771-802. doi: 10.1080/ 00273171.2012.715842

Wang, L. P., Hamaker, E., \& Bergeman, C. S. (2012, 12). Investigating inter-individual differences in short-term intra-individual variability. Psychological Methods, 17(4), 567-581. doi: 10.1037/a0029317

Wang, L. P., \& Maxwell, S. E. (2015, 3). On disaggregating between-person and within-person effects with longitudinal data using multilevel models. Psychological Methods, 20(1), 63-83. doi: 10.1037/met0000030

Warburton, D. E. R., Nicol, C. W., \& Bredin, S. S. D. (2006, 3). Health benefits of physical activity: the evidence. CMAJ : Canadian Medical Association journal, 174(6), 801-9. doi: 10.1503/cmaj.051351

Watson, D., Clark, L. A., McIntyre, C. W., \& Hamaker, S. (1992, 12). Affect, personality, and social activity. Journal of personality and social psychology, 63(6), 1011-25.

Watson, D., Clark, L. A., \& Tellegen, A. (1988, 6). Development and validation of brief measures of positive and negative affect: the PANAS scales. Journal of personality and social psychology, 54(6), 1063-70.

Williams, D. R., \& Mulder, J. (2019). Bayesian Hypothesis Testing for Gaussian Graphical Models: Conditional Independence and Order Constraints.

Williams, D. R., \& Rast, P. (2018). A Bayesian Nonlinear MixedEffects Location Scale Model for Learning. , 1-18.

Williams, D. R., Rast, P., \& Bürkner, P.-C. (2018). Bayesian MetaAnalysis with Weakly Informative Prior Distributions. doi: https://doi.org/10.31234/osf.io/7tbrm

Williams, D. R., Rast, P., Pericchi, L. R., \& Mulder, J. (2019). Comparing Gaussian Graphical Models with the Posterior Predictive Distribution and Bayesian Model Selection. doi: https://doi.org/10.31234/osf.io/yt386

Wilson, K., \& Gullone, E. (1999). The relationship between personality and affect over the lifespan. Personality and Individual Differences, 27(6), 1141-1156. doi: 10.1016/ S0191-8869(99)00058-6

Wilt, J., Noftle, E. E., Fleeson, W., \& Spain, J. S. (2012, 10). The Dynamic Role of Personality States in Mediating the Relationship Between Extraversion and Positive Affect. Journal of Personality, 80(5), 1205-1236. doi: 10.1111/j.1467-6494 .2011.00756.x

Woodrow, H. (1932). Quotidian variability. Psychological Review, 39(3), 245.

Yik, M. S., \& Russell, J. A. (2001, 9). Predicting the Big Two of Affect from the Big Five of Personality. Journal of Research in Personality, 35(3), 247-277. doi: 10.1006/JRPE.2001.2322 